\title{
Exploring the Main Effect of e-Banking on the Banking Industry Concentration Degree on Predicting the Future of the Banking Industry: A Case Study
}

\author{
Zohreh Ghasemi $\mathbb{D}^{1},{ }^{1}$ Mozhdeh Afshar Kermani $\mathbb{D}^{1},{ }^{1}$ and Tofigh Allahviranloo $\mathbb{D}^{2}$ \\ ${ }^{1}$ Department of Applied Mathematics, Tehran North Branch, Islamic Azad University, Tehran, Iran \\ ${ }^{2}$ Faculty of Engineering and Natural Sciences, Bahcesehir University, Istanbul, Turkey \\ Correspondence should be addressed to Mozhdeh Afshar Kermani; mog_afshar@yahoo.com
}

Received 6 June 2021; Accepted 16 August 2021; Published 25 August 2021

Academic Editor: Bekir Sahin

Copyright (c) 2021 Zohreh Ghasemi et al. This is an open access article distributed under the Creative Commons Attribution License, which permits unrestricted use, distribution, and reproduction in any medium, provided the original work is properly cited.

\begin{abstract}
Today, regarding the rapid growth of Information and Communications Technology (ICT), e-commerce, and the subsequent expansion of electronic banking, the banking market structure is also expected to dramatically be changed. This paper aims to explore the main effect of electronic banking on the structure of the Iranian banking industry by investigating the banking market concentration degree. To accomplish this aim, an intelligent hybrid model is developed based on multilayer perceptron neural network and fuzzy regression of the effects of banking on the relative electronic share of banks. In the developed method, the neural network parameters such as weights and errors have been considered as the fuzzy parameters to model it under uncertainty. Ultimately, the descriptive statistics are utilized to evaluate how the difference in relative size of banks concerning e-banking has changed, in addition to exploring the main effect of e-banking on the bank's contribution described as a neural network-fuzzy regression model. Moreover, it shows how the concentration degree in the Iranian banking sector has been reduced. The implemented analysis of the reasons for this decrease reveals that the share of banks has decreased due to an increase in the share of the small banks. Furthermore, model estimation confirms that there exists a positive relationship between banks' share and the use of electronic banking. Besides, the small banks have strongly been shown to utilize the e-banking so that it would lead to an increase in their share and a decrease in the concentration degree. As such, it can be concluded that e-banking has reduced the concentration degree in Iran. The descriptive statistics are employed to prove it.
\end{abstract}

\section{Introduction}

Both economics and industrial organization are the important trends in economics that assess the behavior interaction between producers with each other, producers with customers, and customers with each other. Studying some features such as behavior, structure, and performance of markets is a critical issue of industrial economics, and how these features are related to each other, their causality, and the factors affecting these features have been the subject of several studies in the field of economics. In this matter, the market share is one of the structural variables employed to measure the intensity of competition among producers, which may be affected by performance and behavioral factors in industrial economics literature.
Here, the concentration degree is known as a structural variable that is one of the critical factors affecting the market share of industries, which may have a significant impact on the intensity of banking market competition. In this way, several studies have been conducted to measure the concentration degree of the banking industry, both in Iran and in other countries. Khodadad Kashi and Jafari [1] evaluated the effects of structure of Iranian banking industry. Their results revealed that, during 2000-2010, structural indexes of the Iranian banking industry, including concentration and barriers to entry, have been varied to increase the competitiveness.

Kato and Honjo [2] examined the impact of the concentration degree on the market share instability in 109 Japanese manufacturing industries during 1995-2001. The 
obtained results confirmed that there is a positive and significant relationship between market concentration degree and market share instability [3].

Both e-commerce and e-banking are rapidly expanding and promoting as the novel technology, especially in the information and communication technology around the world. e-banking is one of the main factors that can affect the number, distribution, and market power of both sellers and buyers, or the concentration degree of the banking market. Nevertheless, unfortunately, the impact of this factor on the concentration degree as well as on the intensity of competition (from complete monopoly to complete competition) in the banking market has been paid less attention.

In today's world, employing the quantitative methods to improve financial markets, improving decision making, and investing has become an indispensable necessity. In this regard, the time series prediction is one of the most important predictive areas, in which the past observations of a variable are gathered and then analyzed to understand the fundamental relationships between observations and the determination of a descriptive model. Afterwards, the obtained model is then applied to extrapolate future time series. This modeling approach is very useful and practical especially when there is little available knowledge about the underlying data generation process, or no satisfactory explanatory model that relates the dependent variable to other explanatory variables [4].

Here, the artificial neural network (ANN) is one of the most important nonlinear methods of predicting the series that will provide some suitable results under the proper conditions. The main advantage of this method is the ability of flexible nonlinear modeling. In such networks, there is no need to distinguish the specific shape of the model, and the model is formed based on the information contained in the data. This data-based approach is very useful for many empirical datasets, especially when no theoretical information is available to propose an appropriate data generation process. Despite all the advantages of ANN, these networks contain some disadvantages, based on which the need for large amounts of data to obtain accurate results is one of them.

Although some defined rules for data required for the network have not been regarded, the amount of data required for the network depends on some aspects such as the network structure, the learning algorithm, the complexity of the problem, and the interference in the data. By exploring the impact of different sizes of network data requirements, it can be found that increasing the number of data can optimize the performance of ANN in the prediction problems. It should be noted that as these neural networks can model many complex structures using enough data, they would be more useful than other statistical models for large samples. It should be noted that, however, the ANNs do not require more data to achieve an optimal performance than the linear models. The conducted studies have shown that ANNs contain good predictions with data less than 50 , whereas Box-Jenkins models require at least 50 data for successful predictions [5]. Although the fuzzy prediction methods such as fuzzy regression require less data than other prediction models due to the use of fuzzy numbers instead of deterministic ones, the performance of such methods is not very satisfactory. To avoid the modeling error, fuzzy regression has been proposed that is essentially a spatial prediction model [6,7]; but it also contains some disadvantages, in which the excessive range of interval predictions is the most important of them due to existence of some outliers' values. In this matter, the fuzzy time series are modeled by utilizing both the fuzzy equations and approximate logic [8]. In addition, a time series method is also proposed based on time series and Sang concepts [9]. Utilizing the hybrid models or combining different models is a common approach to improve the accuracy of the predictions. The literature on combinatorial models is very extensive, in which many studies have been carried out since the first research in this field [10-12]. The basic idea of combining multiple models into predictions is to utilize every single model to different patterns in the data. The experimental and theoretical findings indicate that combining different models is an effective and efficient approach to enhance the accuracy of the predictions [13]. The predictive studies have provided several hybrid models using ANN [14].

In this paper, the concentration degree of the Iranian banking industry is investigated using ANN, fuzzy regression, the empirical studies, and the impact of e-banking. The basic concepts of fuzzy logic are utilized to develop a hybrid approach in order to generate better predictions as well as to receive more accurate results, also requiring less data in ANN. In the proposed method, the neural network parameters are considered as the fuzzy numbers instead of deterministic ones. By applying uncertainty environment to the neural networks structure in the form of fuzzy numbers, and taking the advantage of the unique benefits of fuzzy set soft computing, the number of data needed to train and search for possible spaces for optimal parameter values is reduced. Thus, the neural network adjusts its parameters more efficiently when the data available to the network is less than its usual value.

The second section presents the theoretical foundations of the methodology. Section 3 describes the hybrid model based on ANN and fuzzy regression. Section 4 states the use of the hybrid model to investigate how e-banking affects the concentration degree of the Iranian banking industry. At the end, the results are analyzed and conclusions are presented.

\section{Theoretical Principles of Methodology}

This section provides the necessary concepts and backgrounds.

2.1. e-Banking and Services. The transformation of traditional ways of trading and replacing them with e-commerce are of the most important phenomena of the information revolution. Hence, e-banking is the main infrastructure of e-commerce due to the role of money and banking in commerce. Naturally, the role of banks and economic institutions in the transfer of money is crucial. When the Internet showed its business potential along with its 
scientific and research aspects, some business institutions and banks were the first institutions that tried their efforts to utilize this trend in developed countries.

Their efforts are the same as today's e-banking. Then, it quickly clarified that the Internet was a proper tool for a variety of banking and economic activities. Electronic banking has now become a highly specialized, yet highly complex, debate in the world, and its adaptation to commercial and economic policies in different countries requires careful research and planning.

2.2. e-Banking Definitions and Concepts. In the viewpoint of banking system experts, the definition of e-banking is as follows:

(1) Electronic banking can be defined to provide facilities for employees to increase their speed and efficiency in providing on-premises banking services, as well as interbranch and interbank processes around the world, and to provide facilities for customers with hardware and software capabilities that they can perform their desired banking operations securely and with confidence without the need for a physical presence in the bank, through the communication channels every hour of the day.

(2) e-banking is the use of advanced network and telecommunication based software and hardware technologies to exchange resources and financial information electronically, which requires no physical presence at the branch.

(3) Applying information technology and communication platforms (telecommunications) to provide banking services and products to customers as 24/7 services, so that the customer does not need to be present at the branch.

(4) Employing the centralized banking systems, and software and hardware systems to provide banking services to the customer at any time and place.

\subsection{Electronic Banking Products, Services, and Gateways.} In the e-banking discussion, there are three categories as follows: (1) e-banking services, (2) e-banking gateways, and (3) e-banking products, which are closely related to each other. The e-banking gateways are ones that provide e-banking services. Here, two kinds of them are mentioned as follows:

(1) Single-task gateway: it is defined exclusively for e-banking and other undefined services such as automated teller machine (ATM), point of sell (POS), and banking kiosks

(2) Multitask gateway: it is the unlimited banking services such as Internet banking, mobile banking, and telephone banking

Either device or services provided to customers to utilize in e-banking gateways are also called electronic banking products, which can be referred to as bankcard types. At the ends, all banking operations performed by one of the electronic banking gateways or products are called e-banking services.

These mentioned services are generally classified as follows:

(1) Electronic banking terminals and equipment that include the following:
(i) ATM
(ii) Bank kiosk
(iii) Bank card printer
(iv) Branch card reader
(v) POS

(2) Bank cards which include the following:
(i) Debit card
(ii) Gift card
(iii) Credit card
(iv) Debit/credit card
(v) Shopping card
(vi) Prepaid card

(3) Virtual banking systems that include the following:

(i) Mobile banking system

(ii) Telephone banking system

(iii) Internet banking system

(iv) SMS gateway

(v) Electronic payment gateway

(4) Interbank e-banking systems that include the following:

(i) International Bank Account Number (IBAN), which is well known as SHEBA in Iran

(ii) Real-Time Gross Settlement (RTGS), which is well known as SATNA in Iran

(iii) Automated Clearing House (ACH), which is well known as PAYA in Iran

(iv) Script-less Securities Settlement System (SSSS), which is known TABA in Iran

(v) Card-based Electronic Fund Transfer System (CEFTS), which is well known as SAHAB in Iran

2.4. Limer Test and Hausman Test. Occasionally, the collected data we have encountered may be known as both time series and deterministic data. Such a set of data is generally known as a panel data. We have two general cases to estimate the panel data model. The first case is that the $y$-intercept is the same for all the sections, in which we face the pool data model. The second case is the $y$-intercept for all the different sections, which is called panel data model. To identify the above two cases, a test so-called $F$-Limer is carried out.

Therefore, the F-Limer test is utilized to choose between data pool and data panel of regression methods. The statistic of this test is as follows: if the calculated values $F$ are less than the table value, the $H_{0}$ is accepted and only one $y$-intercept should be used. Otherwise, if the calculated $\mathrm{F}$ is greater than the $F$-table, the $H_{0}$ is rejected and the effects of the group are 
accepted, and the different $y$-intercept should be taken into account.

$$
F=\frac{\left(R_{\mathrm{fe}}^{2}-R_{\mathrm{pool}}^{2}\right) /(n-1)}{\left(1-R_{\mathrm{fe}}^{2}\right) /(n t-n-k)}
$$

where $R_{\text {pool }}^{2}$ is the restricted coefficient of determination of regression (the share $y$-intercept), $R_{\mathrm{fe}}^{2}$ is the unrestricted coefficient of determination of regression, $n$ is the number of logical observations, and $k$ is the number of time periods.

Limer (Chao) test: the Limer test is used to choose between a mixed model or a panel model. If the $H_{0}$ is rejected, the mixed model is used and otherwise, it is determined that panel data should be used for modeling. Using the Hausman test, we determine whether to use fixed effects or random effects models. If the $H_{0}$ is rejected, we use the fixed effects model; otherwise, we use the random effects model [3].

Hausman test: the Hausman method is utilized to clarify the effect of a fixed or random model. The fixed effects mean we have the unit $y$-intercept, and in contrast, the random effects will have different values of the $y$-intercept.

F-Limer method: to assess whether variables were used in combination with the F-Limer test. Panel data are analyzed using the $F$-Limer test against the combined data method.

2.5. Herfindahl-Hirschman Competition Index. Several indexes can be employed to estimate the concentration and market share of different firms, such as the inverse of the number of industry firms, the concentration ratio of $k$ firm, the Hannah-Kay index (HKI), the entropy index, Herfindahl-Hirschman index (HHI), the Gini Coefficient, etc. [4]. In terms of theoretical foundations, the Herfindahl-Hirschman index is more robust and reasonable than other indexes. This index utilizes all information from all industry firms. To obtain this index, the sum of squares, the share of production, sales, assets, labor force, and all firms in industry or market are utilized. Indeed, this index weighs each firm as much as its market share and is written as equation (2) (see [15]).

$$
\mathrm{HHI}=\sum_{I=1}^{N}\left(\frac{X_{i}}{\sum_{i=1}^{n} X_{i}}\right)^{2} .
$$

2.6. The Durbin-Watson Test. In regression analysis, especially when variables are evaluated over a time interval, the change data over time may follow a particular pattern. Here, the Durbin-Watson test is performed to detect this pattern. The concept of being independent means that the result of one observation does not affect the result of the other observations. In regression, we often encounter the problem of nonindependent errors when the dependent variable behavior is investigated over a period. Such a relationship in the data is so-called autocorrelation. It should be noted that the linear regression could not be used if the autocorrelation may exist in errors. To test this hypothesis intuitively, graphing the studentized variable sequence in SPSS can be used. Nonetheless, the safer way is to utilize the Durbin-Watson test. Its statistic is between 0 and 4 . If there is no consecutive correlation between the residuals, the value of this statistic should be close to 2 , based on which if it is close to zero, it indicates a positive correlation, and if it is close to 4 , it indicates a negative correlation. Overall, if this statistic is between 1.5 and 2.5 , there is no worry.

The calculation formula is as follows, in which the first order delay is used:

$$
d=\frac{\sum_{t=2}^{T}\left(e_{t}-e_{t-1}\right)^{2}}{\sum_{t=1}^{T} e_{t}^{2}},
$$

where $e_{t}$ is the remainder of $t$ in the regression equation and $T$ is the total number of observations.

2.7. The Concentration Indexes. There are various ways to assess the competitive condition and market structure. These methods are divided into structural and nonstructural methods. The structural methods include the structure-behavior-performance paradigm and the performance structure. The first type considers a strong concentration on the market as a factor in the collusion behavior and performance of the firm. On the other hand, the second type implies the effect of better performance and efficiency on the monopolistic behaviors and consequently the monopoly structure. Investigating a structure based on concentration ratios is a key feature of structural methods. According to some researchers, the size of the market concentration can be estimated using various indexes such as firm concentration ratio, Herfindahl index, reverse number of industry firms, Hannah-Kay index, entropy index, and logarithmic variance and firm size. Among these indices, the Herfindahl-Hirschman index is theoretically more robust and reasonable than other indexes, which is more commonly used to measure the concentration than other indexes. Therefore, Herfindahl-Hirschman concentration index was used.

It is worthwhile to mention that the structural methods also have their categories, in which they are divided into two categories of relative and absolute. The major difference between them is that, in the relative indices, the effect of the number of firms is not taken into account. However, the relative indices can be employed as the appropriate indexes for concentration assessment. The most important relative indexes include Gini coefficient, Linda index, adjusted concentration ratio, and dispersion criterion. In the following, each of these indexes will be introduced in detail. To better understand this concept, consider a concentration curve illustrated in Figure 1.

The concentration curve from the coordinate system is illustrated, where on its horizontal axis is the number of aggregate firms in the market, and on its vertical axis is the aggregate percentage of a particular index of existing firms relative to the aggregate index (e.g., the percentage of aggregate production of those firms relative to total output). On the horizontal axis, firms are classified within the range 


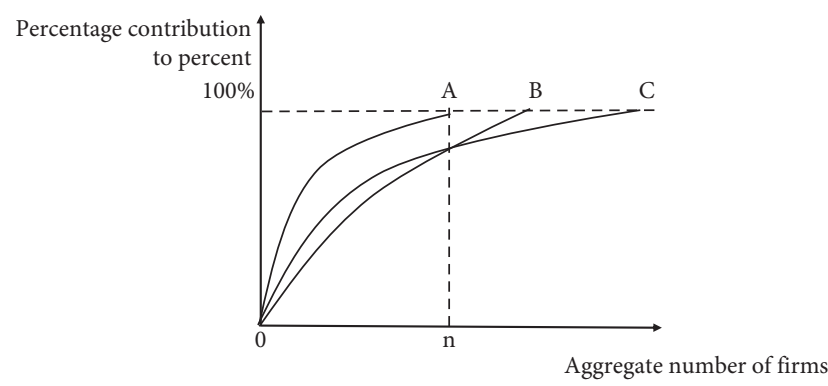

Figure 1: The concentration curve.

of small to large. For this reason, the convexity of the curve is downward, and if all firms are equal in terms of the size, the curve is a straight line. In other words, the convexity of the curve is due to the difference in the size of firms, and the intersection of the concentration curve with the surface represents $100 \%$ of the number of firms in the industry. Hence, it can be said that when the concentration curve of one industry is above and to the left of the concentration of another industry (A), the curve will have a higher concentration degree. However, it is not possible to clearly discuss the intersection of the two concentration curves (B, C), which is illustrated in Figure 1.

2.8. Artificial Neural Networks. ANN is one of the capable and useful methods to estimate multiple nonlinearities in the data and is a flexible computational framework for a wide range of nonlinear problems. Compared to other nonlinear models, one of the clear benefits of such models is that the ANN is a general approximation method and can approximate any function accurately [16]. The strength of neural networks is the parallel processing of data information. It is worthwhile to mention that such networks do not require any assumptions about the shape of the model in the modeling process and are generally a data-based model. Feed forward neural networks with a hidden layer are the most commonly used neural network models to predict time series [7]. Such models include three simple layers of interconnected information processing. The relationship between outputs $\left(y_{t}\right)$ and inputs $\left(y_{t-1}, \ldots, y_{t-p}\right)$ is as follows:

$$
y_{t}=f\left(b_{0}+\sum_{j=1}^{q} \omega_{j} \cdot\left(\sum_{j=1}^{p} \omega_{i, j} \cdot y_{t-i}\right)\right)+\varepsilon_{t},
$$

where $\omega_{j}$ and $\omega_{i, j}$ are model parameters, often called connecting weights; $p$ is the number of input nodes; and $q$ is the number of hidden nodes. $q$ and $f$ are also the activation functions of the middle layer and the output layer, respectively. Sigmoid (relation (4)) and linear functions are some of the functions that are often used as activation functions for hidden and output layers.

$$
\operatorname{Sig}(x)=\frac{1}{1+\exp (-x)} .
$$

The two models of artificial neural networks (equation (4)) will in fact be a nonlinear mapping of past observations to the future value.

$$
y_{t}=f\left(y_{t-1}, \ldots, y_{t-p}, \omega\right)+\varepsilon_{t},
$$

where $\omega$ is the vector of all the parameters and $f$ is the function determined by the neural network structure and the connecting weights. Therefore, the neural network is equivalent to a nonlinear autoregressive model. Equation (6) also states that a neuron in the output layer is utilized for one-step prediction. The simplistic model stated in (6) has a very high approximation power, because if the number of hidden nodes $(q)$ of the bot construction is large enough, it can approximate any arbitrary function. The simple neural-like structure, which has fewer neurons in the hidden layer, often performs better in out-of-sample predictions because of the avoidance of the overfitting problem, mainly in the neural network modeling process. An overfitted model fits well with the data used to construct the model (train dataset), but its development is very poor for out-of-sample data [17]. The number of hidden layer neurons depends on the data, and there are no systematic rules for determining this parameter in the design of neural networks. In addition, another important step in neural network modeling is the selection of the number of interruptions in observations as input network portal, which may be the most important parameter in the design of ANN models, as this parameter plays a crucial role to determine the structure of nonlinear correlations of time series.

There are no specific theories to choose $p$. Therefore, experiences will determine the appropriate value of $p$ and $q$. When the network structure of $p$ and $q$ is specified, the network is ready to train and estimate the parameters. Similar to the modeling autoregressive integrated moving average, the parameters are estimated to minimize the accuracy criterion such as the mean of the squares of errors. The learning process is performed by nonlinear optimization algorithms such as error propagation. The estimated model is usually evaluated by a separate sample that is not used in the learning process (test dataset) and this is different from modeling process of integrated autoregressive moving average models that use a sample to identify, estimate, and evaluate the model. The reason for this is that the general (linear) model is predetermined to model autoregressive integrated moving average [18]. If this is not the case in neural network modeling, and in the ANN modeling, the nonlinear model must be estimated as the model order and the network may be overfitted.

2.9. Fuzzy Regression. The classical regression contains strong implications for the statistical properties of regression models. For instance, the normality or absence of autocorrelation and constant variance are part of the error in the assumption that the violation of any of these assumptions invalidates the results of classical regression analysis. In most cases, it is difficult to justify these assumptions or in some cases, they cannot be used properly. For example, either observations or definitions of a system may affect human or inaccurate estimates and judgments, and inadequate information in the use of variables. In general, although classical regression has many uses, it will be misleading in the following situations: 
(1) There are insufficient observational data

(2) Errors do not follow the normal distribution

(3) The relationship between independent and dependent variables is ambiguous

(4) There is ambiguity about an event

(5) There are incorrect linearization assumptions

In such cases where the classical regression methodology and its hypotheses are difficult to justify, the use of fuzzy regression with membership probability distribution for inaccuracy or ambiguity may increase our understanding of the system and the results [19]. Here, there are several models of fuzzy regression. In this paper, fuzzy regression model was developed, which achieves the best regression equation by minimizing fuzziness. In order to achieve a good fit, an optimization model must be developed. Regarding the fact that the membership functions used to represent fuzzy numbers are triangular, fuzzy regression can be formulated as a linear programming problem. The fuzzy likelihood regression model used in this study is a model where the input and output are nonfuzzy numbers, but the computational parameters are fuzzy numbers.

The membership function of the coefficients of the regression model is in the form of symmetric triangular fuzzy numbers, which is defined as follows:

$$
\mu_{\beta_{i}}\left(\beta_{i}\right)= \begin{cases}1-\frac{\left|\alpha_{i}-\beta_{i}\right|}{c_{i}}, & \alpha_{i}-c_{i} \leq \beta_{i} \leq \alpha_{i}+c_{i}, \\ 0, & \text { otherwise, }\end{cases}
$$

where $\mu_{\beta_{i}}\left(\beta_{i}\right)$ is a membership function of the fuzzy set and denotes the regression coefficients. In the above relation, $\alpha_{i}$ and $c_{i}$, respectively, are the center and width of the membership function, so $\widetilde{A}_{i}=\left(\alpha_{i}, c_{i}\right)$ [20]. Therefore, the regression equation can be written as follows:

$$
\tilde{Y}=\widetilde{\beta}_{0}+\widetilde{\beta}_{1} X_{1}+\cdots+\widetilde{\beta}_{n} X_{n}+\varepsilon .
$$

$$
E(p)=\operatorname{Min}\left(b_{0 \alpha} u_{t, 0}+\omega_{1 \alpha} u_{t, 1}+\cdots+\omega_{q \alpha} u_{t, q}-y_{\alpha t}\right)^{2}+\frac{1}{6}\left(b_{0 c} u_{t, 0}+\omega_{1 c}\left|u_{t, 1}\right|+\cdots+\omega_{q c}\left|u_{t, q}\right|-y_{c t}\right)^{2}
$$

The steps of the hybrid method are as follows:

(i) First step: train a neural network model using the information contained in the observations (which are nonphase). The result of the first step is the optimal solution of the parameter $\omega^{*}=\left(\omega_{0}^{*}, \omega_{1}^{*}, \ldots, \omega_{q}^{*}\right)$ which is used as one of the input datasets in the second step.

(ii) Second step: determine the minimum ambiguity using equation (10) and the weights obtained from the first step are $\omega^{*}=\left(\omega_{0}^{*}, \omega_{1}^{*}, \ldots, \omega_{q}^{*}\right)$. The number of constraints is equal to the number of
2.10. Formulating a Hybrid Model. As mentioned before, although ANNs are accurate prediction models for a wide range of problems, they contain some restrictions for a large amount of past data to obtain accurate results, whereas today it is often necessary to predict future situations using low data in short-run times due to the uncertainty of the environment and the rapid development of new technologies. Therefore, in today's world, predictive methods are required that need fewer data and are efficient in such situations. The fuzzy regression model is a good base prediction model under low data conditions, but does not always perform well. The aim of this paper is to demonstrate the advantages of fuzzy regression to predict time series and to overcome the limitations of the need for large data in ANN method for prediction.

The neural network parameters (weights and bias) are deterministic values $b_{0}, b_{0, j}, \omega_{j}$, and $\omega_{i, j}$ whereas in the proposed method, instead of applying these definitive values, the factors are considered as triangular fuzzy numbers $\left(\widetilde{\omega}_{j}, \widetilde{b}_{0}\right)$.

$$
\widetilde{y}_{t}=\widetilde{b}_{0}+\sum_{j=1}^{q} \widetilde{\omega}_{j} \cdot g\left(b_{0, j}+\sum_{j=1}^{p} \widetilde{\omega}_{i, j} \cdot y_{t-i}\right),
$$

where $\tilde{y}_{t}$ are the observations and $\tilde{b}_{0}, \widetilde{\omega}_{j}$ are fuzzy numbers. Now equation (9) by converting $u_{t, j}=\left(b_{0, j}+\sum_{j=1}^{p} \omega_{i, j} \cdot y_{t-i}\right)$ is transformed as follows:

$$
\tilde{y}_{t}=\widetilde{b}_{0}+\sum_{j=1}^{q} \widetilde{\omega}_{j} \cdot u_{t, j}
$$

The fuzzy parameter in this equation is given as triangular fuzzy numbers in accordance with (4). Assume $\widetilde{y}_{t}=\left(y_{\alpha t}, y_{c t}\right)$, so that $y_{\alpha t}, y_{c t}$ are defined as follows:

$$
\begin{aligned}
& y_{\alpha t}=b_{0 \alpha} u_{t, 0}+\omega_{1 \alpha} u_{t, 1}+\cdots+\omega_{q \alpha} u_{t, q}+\varepsilon_{1} \text {, } \\
& y_{c t}=b_{0 c} u_{t, 0}+\omega_{1 c}\left|u_{t, 1}\right|+\cdots+\omega_{q c}\left|u_{t, q}\right|+\varepsilon_{2} \text {, } \\
& \text { for } j=0,1, \ldots, q, t=1,2, \ldots, k \text {. }
\end{aligned}
$$

If $\omega_{j, \alpha}$ and $\omega_{j, s}$ are the weights of the relation between the output neuron and the $j^{\text {th }}$ layer neuron of the middle layer, 
dataset contains distinct differences or out-ofbounds.

2.11. Methodology. The current research is an application paper and quantitative in terms of the applied method. The method used in this study is to calculate concentration and econometric indexes. The concentration degree will be measured using the Herfindahl-Hirschman index and the hybrid fuzzy regression neural network method is used to investigate the effect of e-banking on the concentration degree. The variables studied in the paper are as follows:

(i) Banks' share of bank deposit

(ii) Number of ATMs separated by a bank

(iii) Number of POSs separated by a bank

(iv) Number of PINPADs separated by a bank

The data used in the study are data and are a chart of the period 2010-2018 of the data used in the study. The statistical population of the study includes 13 Iranian banks. The method of data gathering is libraries (databases) spatial research area, Iranian banking, and research area of Iranian banks. Since the main purpose of the present study is to examine the structure of the Iranian banking industry and determine the severity of the impact of e-banking on the concentration degree of this industry.

It is necessary to study in two parts:

(1) In the first part, different types of market structure measurement indexes are evaluated and the most appropriate one is related to the Iranian banking industry. Then, the effect of e-banking on the concentration degree of each existing bank in this industry is analyzed and a mathematical model is developed from the results.

(2) In the second part, we also estimate the effect empirically.

\section{Introduction of Fuzzy Regression Model}

The initial fuzzy regression equation will be as follows:

$$
D_{t}^{i}=\tilde{\beta}_{0}+\tilde{\beta}_{1} \mathrm{IT}_{t}^{i}+\varepsilon_{t}^{i} .
$$

In other words, if the $\widetilde{\beta}_{1}$ center is a positive and significant number, it means that e-banking has attracted more deposits for each bank and increased the bank's share. Nevertheless, if the $\widetilde{\beta}_{1}$ center is negative and significant, it denotes that e-banking has eliminated more deposits and reduced its share of the bank, based on which there may be a dual effect of supply and demand on network effects. This has a negative impact on the bank's share, which may reduce the consumer impact through adverse consumer information. Therefore, if the relationship between market share and e-banking is positive, it indicates a network effect; but if this relationship is negative, no definitive conclusion can be stated about its cause.

Here, we consider the three input channels to examine the effect of e-banking on market concentration on deposits viewpoint:

(i) ATM

(ii) PINPAD

(iii) $\mathrm{POS}$

(iv) Moreover, NB and RI represent the number of branches and the rate of interest, respectively. They are also the controlling variable.

$$
D_{t}^{i}=\widetilde{\alpha}_{0}+\widetilde{\alpha}_{1} \mathrm{ATM}_{t}^{i}+\widetilde{\alpha}_{2} \mathrm{PINPAD}_{t}^{i}+\widetilde{\alpha}_{3} \operatorname{POS}_{t}^{i}+\widetilde{\alpha}_{4} \mathrm{NB}_{t}^{i}+\widetilde{\alpha}_{5} \mathrm{RI}_{t}^{i}+\widetilde{\varepsilon}_{t}^{i}
$$

As the Herfindahl and Hirschman index discusses the concentration degree on the relative size of banks, it is necessary to compute and enter variables as a ratio:

$$
\frac{d_{t}^{i}}{D_{t}^{T}}=\widetilde{\alpha}_{0}+\alpha_{1} \frac{\mathrm{ATM}_{t}^{i}}{\mathrm{ATM}_{t}^{T}}+\widetilde{\alpha}_{2} \frac{\operatorname{PINPAD}_{t}^{i}}{\operatorname{PINPAD}_{t}^{T}}+\widetilde{\alpha}_{3} \frac{\operatorname{POS}_{t}^{i}}{\operatorname{POS}_{t}^{T}}+\widetilde{\alpha}_{4} \frac{\mathrm{NB}_{t}^{i}}{\mathrm{NB}_{t}^{T}}+\widetilde{\alpha}_{5} \mathrm{RI}_{t}^{i}+\varepsilon_{t}^{i}
$$

where $d_{t}^{i} / D_{t}^{T}$ is the ratio of each bank's deposit to the total bank deposit in year $t$ (which is fuzzy valuation), $\mathrm{ATM}_{t}^{i} / \mathrm{ATM}_{t}^{T}$ is the ratio of the number of ATMs in each bank to the total number of ATMs in year $t$, $\mathrm{PINPAD}_{t}^{i} / \mathrm{PINPAD}_{t}^{T}$ is the ratio of the number of PINPADs in each bank to the total number of PINPADs in year $t$, $\operatorname{POS}_{t}^{i} / \operatorname{POS}_{t}^{T}$ is the ratio of the number of POSs of each bank to the total number of banks' POSs in year $t, \mathrm{NB}_{t}^{i} / \mathrm{NB}_{t}^{T}$ is the ratio of the number of branches of each bank to the total branches of banks in year $t$, and $\mathrm{RI}_{t}^{i}$ is the specified interest rate (separately for government and private banks) for each bank in year $t$.

Therefore, the regression equation of relation (16) can be rewritten as follows and relation (11) as follows: 


$$
\frac{d_{t}^{i}}{D_{t}^{T}}=\left(\alpha_{0}, s_{0}\right)+\left(\alpha_{1}, s_{1}\right) \frac{\mathrm{ATM}_{t}^{i}}{\mathrm{ATM}_{t}^{T}}+\left(\alpha_{2}, s_{2}\right) \frac{\mathrm{PINPAD}_{t}^{i}}{\operatorname{PINPAD}_{t}^{T}}+\left(\alpha_{3}, s_{3}\right) \frac{\operatorname{POS}_{t}^{i}}{\operatorname{POS}_{t}^{T}}+\left(\alpha_{4}, s_{4}\right) \frac{\mathrm{NB}_{t}^{i}}{\mathrm{NB}_{t}^{T}}+\left(\alpha_{5}, s_{5}\right) \mathrm{RI}_{t}^{i}+\varepsilon_{t}^{i},
$$

so that $\alpha$ and $s$ are the vectors of the values of the factors and their widths around the center, respectively. We write for ease of work

$$
\tilde{Y}_{i}^{t}=\left(\alpha_{0}, s_{0}\right)+\left(\alpha_{1}, s_{1}\right) A_{t}^{i}+\left(\alpha_{2}, s_{2}\right) \mathrm{PI}_{t}^{i}+\left(\alpha_{3}, s_{3}\right) \mathrm{PO}_{t}^{i}+\left(\alpha_{4}, s_{4}\right) N_{t}^{i}+\left(\alpha_{5}, s_{5}\right) R_{t}^{i}+\varepsilon_{t}^{i},
$$

where

$$
\left\{\begin{array}{l}
\widetilde{Y}_{i}^{t}=\frac{d_{t}^{i}}{D_{t}^{T}}, \\
A_{t}^{i}=\frac{\operatorname{ATM}_{t}^{i}}{\mathrm{ATM}_{t}^{T}}, \\
\mathrm{PI}_{t}^{i}=\frac{\operatorname{PINPAD}_{t}^{i}}{\operatorname{PINPAD}_{t}^{T}} \\
\mathrm{PO}_{t}^{i}=\frac{\operatorname{POS}_{t}^{i}}{\operatorname{POS}_{t}^{T}}, \\
N_{t}^{i}=\frac{\mathrm{NB}_{t}^{i}}{\mathrm{NB}_{t}^{T}}, \\
R_{t}^{i}=\mathrm{RI}_{t}^{i} .
\end{array}\right.
$$

It should be noted that these mentioned variables are applied without referring to their ratio to facilitate for calling the name of variables.

3.1. The Predictive Evaluation Indexes. In this paper, an optimization technique is utilized to minimize the neural network objective function. Thus, we use equation (10) to measure the prediction accuracy of the neural network objective function represented by the symbol $E(p)$. The closer the value of $E(p)$ to zero, the greater the prediction accuracy. On the other hand, by choosing the objective function introduced in equation (10) and training the neural network, the neural network weights are obtained that can be considered as the regression coefficients.

3.2. Decision-Making. As the regression coefficients of the model are fuzzy numbers, the output variable is fuzzy. Therefore, in order to be used in decision-making and comparison, it must be converted to a nonfuzzy number. The process by which each fuzzy set is assigned a number is called "defuzzification," where there exist several ways to apply it.
In this paper, the center of gravity method is used, which is defined in the following equation:

$$
A=\frac{\int \mu(x) x \mathrm{~d} x}{\int \mu(x) \mathrm{d} x} .
$$

3.3. The Estimation Model. The model is considered in the period 2010-2018. We first estimate the regression model using Eviews software. The first principle in estimating the regression model is to study the stationability of the variables. Regarding the fact that most macroeconomic time series variables are nonstationary, if the longtime relationship between the model variables is not investigated, an estimated regression may be false, and therefore, it is necessary to investigate the longtime relationship between the model variables. For this purpose, we use unit root test and summary pattern.

In this study, the summary model is employed because it is general and includes other types of stationability tests. Particular attention is paid to Levin, Lin, and Chut method for the analysis of studying the stationability of the variables in summary pattern. Table 1 reports the unit root test on the variables of ATM, PINPAD, f POS, NB, and RI. For all these variables, a test was performed. For all variables, based on the $\mathrm{H}_{0}$ and the probability obtained, the existence of a unit root is rejected and the unit root variable does not exist, which means that all variables are stationary. The information from this test is listed in Table 1.

Now, regarding the stationability of all the variables, we can estimate in the panel environment. Of course, it is necessary to check whether the model is pool or panel, and whether the effects are fixed or random. Thus, first, by using the $F$-Limer test, we examine the panel model or pool data, and then by using the Hausman test, we evaluate the model's fixed or random effects. The $F$-Lemer and Hausman test was performed on a model consisting of variables of ATM PINPAD, POS, NB, and RI. The experimental results are provided in Table 2 . Considering the probability value of the $F$-Limer test, $H_{0}$ is rejected and the panel model for pool is confirmed. Besides, regarding the probability of the Hausman test, the $H_{0}$ is rejected and the fixed effects model with random effects is confirmed.

Therefore, the reliability of the stationability variables is obtained and we know that we have a panel model with fixed 
TABLE 1: Investigation of stationability of variables.

\begin{tabular}{lcccc}
\hline Variable & Probability & Statistics value & Method & Status \\
\hline PINPAD & 0.00001 & -13.6 & Levin, Lin, and Chut & $H_{0}$ is rejected (lack of unit root) \\
POS & 0.00001 & -11.6 & Levin, Lin, and Chut & $H_{0}$ is accepted (lack of unit root) \\
NB & 0.00001 & -5.3 & Levin, Lin, and Chut & $H_{0}$ is rejected (lack of unit root) \\
RI & 0.0041 & -2.6 & Levin, Lin, and Chut & $H_{0}$ is rejected (lack of unit root) \\
ATM & 0.00001 & -10.6 & Levin, Lin, and Chut & $H_{0}$ is rejected (lack of unit root) \\
Deposit & 0.00001 & -5.9 & Levin, Lin, and Chut & $H_{0}$ is rejected (lack of unit root) \\
\hline
\end{tabular}

References: results of this study.

TABLE 2: Results of the F-Limer and Hausman tests.

\begin{tabular}{lcccc}
\hline Test & $\begin{array}{c}\text { Probability } \\
\text { value }\end{array}$ & $\begin{array}{c}\text { Statistics } \\
\text { value }\end{array}$ & $\begin{array}{c}\text { Degrees of } \\
\text { freedom }\end{array}$ & Description \\
\hline $\begin{array}{l}\text { F-Limer test } \\
\begin{array}{l}\text { Hausman } \\
\text { test }\end{array}\end{array}$ & 0.00001 & 47.08 & 19.100 & $\begin{array}{c}H_{0} \text { is rejected (panel model approval related to pool) } \\
H_{0} \text { is rejected (confirmation of fixed effects model related to random } \\
\text { effects) }\end{array}$ \\
\hline
\end{tabular}

References: results of this study.

effects. Now for estimation, we have ATM, PINPAD, POS, $\mathrm{NB}$, and RI variables as well as deposits and we make initial estimates. Since the coefficients obtained are fuzzy numbers and this fuzzy number is obtained from the cassette and adding a wide value to the central value, it will be interpreted as complete. To solve this problem, we use equation (20) to defuzzify the estimated defuzzification coefficient values. The results of the estimation are described in Table 3.

The Durbin-Watson numerical value and $R^{2}$ are (1.0170) and (0.9826), respectively. Regarding the Durbin-Watson numeric value, it can be said that the model suffers from autocorrelation. For this purpose, we insert AR (1) into the model. We also estimated the model in different scenarios with the above variables in mind. The best case scenario was the model with the interrupted ATM variable. That is, the probability values were significant and the Akaike and Schwarz criteria were at their lowest possible. However, to be sure, we also used interrupts 1 or 2 for the ATM variable, the interrupt structure test, and the interrupt 2 relative to the interrupt 1 was confirmed for the ATM variable. Thus, we set interrupt 1 for the ATM variable, as represented in Table 4.
Watson's numerical Durbin and $R^{2}$ are (2.14) and (0.99), respectively. Regarding the coefficient of determination $R^{2}$, it can be said that the regression equation estimates up to $0.99 \%$ of deposit changes. Also, given the size of the Durbin-Watson, which is close enough to the number 2, we can say that we face autocorrelation. It means the time errors $t$ and $t-1$ are independent. As the probability value of the RI variable is still not significant, it should be excluded from the model. Therefore, we consider the coefficients of the remaining variables as the initial weights of the neural network and train the neural network. The results are presented in Table 5.

The Durbin-Watson numerals (network output center and deposition center) are (2.17) and $R^{2}(0.99)$. As mentioned in the previous table, the Durbin-Watson rate and the coefficient of determination $\left(R^{2}\right)$ are suitable numbers, and the same interpretations can be considered. Now to obtain the approximation, we had better consider the coefficients obtained in Table 5, as the initial weights of the fuzzy regression neural network model as well as train the neural network to obtain the optimal weights, in which our proposed model is achieved as follows:

$$
\begin{gathered}
Y_{i}^{t}=\left(\alpha_{0}, s_{0}\right)+\left(\alpha_{1}, s_{1}\right) A_{t}^{i}+\left(\alpha_{2}, s_{2}\right) \mathrm{PI}_{t}^{i}+\left(\alpha_{3}, s_{3}\right) \mathrm{PO}_{t}^{i}+\left(\alpha_{4}, s_{4}\right) N_{t}^{i}+\varepsilon_{t}^{i}, \\
Y_{i}^{t}=(0.026,0.0000)+(-0.208,0.0003) A_{t}^{i}+(0.123,0.0001) \mathrm{PI}_{t}^{i} .
\end{gathered}
$$

The neural network is trained by the ordinary least squares error method. In the general form, the equivalent of regression, $\alpha_{0}$ is equal to the mean value of the dependent variable when the explanatory variable is zero. It is also stated here that $\left(\alpha_{0}, s_{0}\right),\left(0.026, s_{0}\right)$ is equal to the average value of the deposit share when the explanatory variables are zero. Moreover, $\left(\alpha_{1}, s_{1}\right),\left(\alpha_{2}, s_{2}\right),\left(\alpha_{3}, s_{3}\right),\left(\alpha_{4}, s_{4}\right)$ indicate an average increase in the share of deposits per unit increase in the NB, the number of POSs, the number of PINPADs, and the number of ATMs and RI. Estimates show that ATMs have a negative effect, and PINPADs and POSs have a positive effect on bank deposit share. Overall, it can be said that e-banking has a positive effect on the deposit ratio of banks. Also, the positive coefficient of variation of the NB has been obtained which indicates that as the NB increases, the bank will have more deposit share.

In addition, no stability tests were performed due to the panel model and the use of Eviews software. This is because, according to Eviews software, this test is not possible in the panel environment. It should be noted that panel outputs are 
TABLE 3: The obtained estimations.

\begin{tabular}{lccc}
\hline Variable & Probability value & $t$ statistics & The central value of the coefficients \\
\hline PINPAD & 0.024 & 2.28 & 0.083 \\
POS & 0.264 & -1.12 & -0.018 \\
NB & 0.003 & 3.03 & 0.535 \\
RI & 0.393 & 2.28 & 0.083 \\
ATM & 0.324 & -1.12 & -0.018 \\
\hline
\end{tabular}

References: results of this study.

TABle 4: The obtained estimations for the coefficients.

\begin{tabular}{lccc}
\hline Variable & Probability value & $t$ statistics & The central value of the coefficients \\
\hline PINPAD & 0.0051 & 2.9217 & 0.1453 \\
POS & 0.0215 & 2.3694 & 0.1231 \\
NB & 0.0234 & 2.3344 & 0.7585 \\
RI & 0.5672 & 0.5757 & 7.34 \\
ATM & 0.0008 & -3.5676 & -0.262 \\
AR $(1)$ & 0.0963 & 1.6931 & 0.2572 \\
\hline
\end{tabular}

References: results of this study.

TABLE 5: The obtained values for the coefficients.

\begin{tabular}{lccc}
\hline Variable & Probability value & $t$ statistics & The central value of the coefficients \\
\hline PINPAD & 0.0154 & 2.4813 & 0.12 \\
POS & 0.0088 & 2.6900 & 0.12 \\
NB & 0.069 & 1.8411 & 0.46 \\
RI & 0.0049 & -2.9023 & -0.207 \\
ATM & 0.0022 & 3.1789 & 0.373 \\
AR(1) & 0.0154 & 2.4813 & 0.12 \\
\hline
\end{tabular}

References: results of this study.

typically used to obtain the variables' relationships and the intensity of the variables' relationships.

As discussed before, the aim of this paper is to explore the main effect of e-banking on the concentration degree, while the above relationship, i.e., the positive relationship between e-banking and bank deposit share, does not explain how the industry's concentration (deposit viewpoint) has changed. Discussion on how e-banking has an impact on the concentration degree, in addition to the knowledge of the above relationship, i.e., the positive relationship between e-banking and bank deposit contributions, requires descriptive statistics and data analysis. If, however, there is an increase in the market share of the bank (because of e-banking deduced from the analysis), the degree of concentration in the banking industry would increase due to the increase in the relative size of existing banks.

But if the market shares of banks increases toward reducing the relative size of existing banks, the concentration degree in the banking industry would decline as well. As such, to expand the effect of e-banking on the concentration degree of the Iranian banking sector, or in other words, to assess how the relative size of banks changes with respect to electronic banking, in addition to examining the impact of electronic banking on banks' contribution as a hybrid model of neural network-fuzzy regression, the descriptive statistics are also employed. This is carried out using Excel software. In general, it should be clear to what extent either the increase or decrease in the banks' share has been beneficial to degree concentration on either increasing or decreasing the concentration.

\section{Descriptive Statistics and Data Analysis}

4.1. Ranking and Categorization of Iranian Banks. In the descriptive statistics and data analysis section, we first need to divide the existing banks into three small, medium, and large divisions, based on the amount of deposits they have with other banks, because we need this segmentation for further work and further investigation. The following steps are used to determine the size and rating of Iranian banks:

(1) We obtain the average of the ratio of the deposit of each bank to the total deposit of each bank (years 2010-2018) (year $t=d_{t}^{i} / D_{t}^{T}$ ) during years 1-9

(2) We divide the banks into three categories: large, medium, and small

The results are presented in Table 6.

According to the second column of Table 6, it can be said that during the years 2010-2018, on deposit viewpoint, the banks numbered from 1 to 21 , respectively, have accounted for the highest to the lowest market share.

We now need to divide the banks into three categories:

(1) Large banks: banks with more than 10\% market share, named as Banks 1 to 4 
TABLE 6: Ranking of Iranian banks.

\begin{tabular}{|c|c|}
\hline Iranian banks & Average deposit ratio of each bank to total bank deposits during the years 2010-2018 \\
\hline Bank 1 & 0.175 \\
\hline Bank 2 & 0.146 \\
\hline Bank 3 & 0.115 \\
\hline Bank 4 & 0.100 \\
\hline Bank 5 & 0.052 \\
\hline Bank 6 & 0.050 \\
\hline Bank 7 & 0.041 \\
\hline Bank 8 & 0.040 \\
\hline Bank 9 & 0.034 \\
\hline Bank 10 & 0.028 \\
\hline Bank 11 & 0.020 \\
\hline Bank 12 & 0.0160 \\
\hline Bank 13 & 0.0140 \\
\hline Bank 14 & 0.0134 \\
\hline Bank 15 & 0.1210 \\
\hline Bank 16 & 0.0120 \\
\hline Bank 17 & 0.0080 \\
\hline Bank 18 & 0.0059 \\
\hline Bank 19 & 0.0036 \\
\hline Bank 20 & 0.0028 \\
\hline Bank 21 & 0.0023 \\
\hline Total & 1 \\
\hline
\end{tabular}

References: results of this study.

(2) Medium bank: banks between 1\% and 10\% market shares, named as Banks 5 to 15

(3) Small banks: banks with less than 1\% market share, named as Banks 16 to 21

4.2. Descriptive Statistics. As mentioned, to explore the impact of e- banking on the concentration degree of the Iranian banking industry, in addition to know how electronic banking is affecting on share market (discussed earlier) we need to know how banks experience an increase or decrease in the share due to e-banking. The following six modes can be considered:

(i) Large banks have a higher share

(ii) Medium banks have a higher share

(iii) Medium banks have a lower share

(iv) Small banks have a lower share

If the decline is due to the increased market share of banks in order to increase the difference in the relative share of existing banks, then the concentration on the banking industry will increase. On the other hand, if this decline is due to the increased market share of banks in order to reduce the difference in the relative share of existing banks, the concentration degree in the banking industry will decrease. For instance, if the decline in the shareholding of large banks is stronger than one of small- and medium-sized banks, the difference in the relative size of existing banks will be reduced and as a result the concentration of industry would be reduced.

Nevertheless, if this decline in shareholder performance is stronger for small banks, the difference will increase in the relative size of existing banks, and the concentration degree in the industry will increase. Furthermore, if the increase in the shareholding of large banks is stronger than one of small- and medium-sized banks, the difference will increase in the relative size of existing banks, and the concentration degree in the industry will increase. However, if the increase in the shareholding of small banks becomes stronger, the discrepancy in the relative size of existing banks is reduced and as a result the concentration degree on the industry would be reduced.

Of course, based on the results of the regression model, i.e., the positive relationship between e-banking and banks' share (deposit viewpoint) as well as the benefit of all existing banks in the sample of e-banking, one can only say that the problem of increasing banks' share is being faced. Now, the main question will be raised whether large banks are experiencing more growth, or medium banks or small banks.

Therefore, what we need to answer is how to grow the benefits of e-banking as well as how to grow deposits among banks. After knowing how to categorize the size of banks, the average annual growth rate of e-banking benefits and average growth rate is required to know how many banks experience most of this increase in the share of e-banking and deposits. The average annual growth rate of deposits for three groups of large, medium, and small banks is provided in Table 7.

Now, to better evaluate and compare the three sets of large, medium, and small banks, we need to denote the average annual growth rate of the deposit, as well as the average growth rate of the electronic banking benefit for all three sets of bank size in numerical form, which is clearly observed in Table 8.

The average growth rate of e-banking benefit and subsequently (due to the positive correlation implied by the regression model) the higher average deposit growth rate in 
TABLE 7: Utilization growth of electronic banking and deposit in banks.

\begin{tabular}{|c|c|c|c|c|}
\hline $\begin{array}{l}\text { Bank category } \\
\text { (deposit } \\
\text { viewpoint) } \\
\end{array}$ & $\begin{array}{c}\text { Average of annual growth of } \\
\text { utilization from ATMs } \\
\text { during 2010-2018 (\%) }\end{array}$ & $\begin{array}{c}\text { Average of annual growth of } \\
\text { utilization from PINPADs } \\
\text { during 2010-2018 (\%) }\end{array}$ & $\begin{array}{l}\text { Average of annual growth of } \\
\text { utilization from POSs during } \\
2010-2018(\%) \\
\end{array}$ & $\begin{array}{c}\text { Average of annual } \\
\text { growth of deposits } \\
\text { during 2010-2018 (\%) }\end{array}$ \\
\hline \multicolumn{5}{|l|}{ Large banks set } \\
\hline Bank 1 & 25.3 & 102.7 & 25.8 & 17.8 \\
\hline Bank 2 & 15.8 & 72.8 & 12.2 & 21.4 \\
\hline Bank 3 & 15.6 & 47.3 & 13.2 & 20.9 \\
\hline Bank 4 & 22.8 & 62.07 & 15.3 & 22.9 \\
\hline \multicolumn{5}{|l|}{$\begin{array}{l}\text { Medium banks } \\
\text { set }\end{array}$} \\
\hline Bank 5 & 25.9 & 24.3 & 8.9 & 18.5 \\
\hline Bank 6 & 11.2 & 44.3 & -2.7 & 13.1 \\
\hline Bank 7 & 40.6 & 49.4 & 28.8 & 18.7 \\
\hline Bank 8 & 18.1 & 33.09 & 27.2 & 17.5 \\
\hline Bank 9 & 34.8 & 118.26 & 9.5 & 46.5 \\
\hline Bank 10 & 20.9 & 64.19 & 24.1 & 30.5 \\
\hline Bank 11 & 19.8 & 95.9 & 9.9 & 26.8 \\
\hline Bank 12 & 35.4 & 27.3 & 61.2 & 29.9 \\
\hline Bank 13 & 49.5 & 124.3 & 12.8 & 27.2 \\
\hline Bank 14 & 20.4 & 293.09 & 8.9 & 29 \\
\hline Bank 15 & 26.1 & 64.19 & 8.9 & 22.2 \\
\hline \multicolumn{5}{|l|}{ Small banks set } \\
\hline Bank 16 & 97.6 & 121.5 & 104.3 & 73.7 \\
\hline Bank 17 & 36.8 & 156.7 & 6.04 & 58.9 \\
\hline Bank 18 & 7.1 & -5.78 & 8.7 & 19.5 \\
\hline Bank 19 & 15.8 & 0.98 & 4.6 & 33.8 \\
\hline Bank 20 & 51.9 & 202.4 & 118.9 & 35.46 \\
\hline Bank 21 & 0.09 & 45.2 & -23.01 & 151.5 \\
\hline
\end{tabular}

References: results of this study.

TABLE 8: Average growth among banks.

\begin{tabular}{lccccc}
\hline $\begin{array}{l}\text { Bank category } \\
\text { (deposit } \\
\text { viewpoint) }\end{array}$ & $\begin{array}{c}\text { Average of annual } \\
\text { growth of } \\
\text { utilization from } \\
\text { ATMs (\%) }\end{array}$ & $\begin{array}{c}\text { Average of annual } \\
\text { growth of utilization } \\
\text { from PINPADs (\%) }\end{array}$ & $\begin{array}{c}\text { Average of annual } \\
\text { growth of } \\
\text { utilization from } \\
\text { POSs (\%) }\end{array}$ & $\begin{array}{c}\text { Average of } \\
\text { annual growth } \\
\text { of deposits (\%) }\end{array}$ & Description \\
\hline $\begin{array}{l}\text { Large banks set } \\
\text { sedium bank }\end{array}$ & 19.8 & 71.2 & 16.6 & 20.7 & $\begin{array}{c}\text { Small- and medium-sized banks, } \\
\text { respectively, have the highest average } \\
\text { annual growth rate of deposit share, } \\
\text { as well as the average growth rate of } \\
\text { ATMs, POSs, and PINPADs. }\end{array}$ \\
\hline
\end{tabular}

References: results of this study.

the market are observed. Regarding the results of the above tables, small banks have experienced an increase in e-banking profitability and subsequently more deposit growth. In other words, the relative size of existing banks has diminished, and the concentration degree on the industry is shrinking. Therefore, e-banking can reduce the concentration degree of the banking industry in Iran. In order to ensure the accuracy and inconsistency of this conclusion with the reality of the degree trend in the Iranian banking industry, we investigate the concentration degree in the industry. Before analyzing the concentration degree in the Iranian banking industry, we will have another analysis of the available data. Looking at the issue from this aspect (which we will go on to say) is not without merit to predict the process of concentration in Iran.
As Table 9 shows, the data on the growth rate of e-banking benefit, and the deposit growth data on small bank sets, are more dispersed than the same data on medium and large bank sets. This reveals that, despite the overall good growth in deposits and the benefits of electronic banking for small banks, not all banks in this portfolio have the same performance, and the distribution of banks is high. In other words, only the very large growth in deposits and the benefits of e-banking in some of the banks in the small bank complex have led to a higher growth rate than the medium and large banks. In fact, if this performance extends to other banks in this portfolio, assuming the stability of other conditions, we will soon see further growth of this portfolio, and a further decline in the concentration degree. 
TABLE 9: Dispersion in average growth of bank sets.

\begin{tabular}{lcccc}
\hline $\begin{array}{l}\text { Bank category } \\
\text { (deposit } \\
\text { viewpoint) }\end{array}$ & $\begin{array}{c}\text { Variation coefficient of } \\
\text { average growth of utilization } \\
\text { from ATMs (\%) }\end{array}$ & $\begin{array}{c}\text { Variation coefficient of } \\
\text { average growth of utilization } \\
\text { from PINPADs (\%) }\end{array}$ & $\begin{array}{c}\text { Variation coefficient of } \\
\text { average growth of utilization } \\
\text { from POSs (\%) }\end{array}$ & $\begin{array}{c}\text { Variation coefficient of } \\
\text { average annual growth } \\
\text { (\%) }\end{array}$ \\
\hline Large banks set & 21.6 & 28.5 & 32.7 & 9.7 \\
Medium banks set & 39.2 & 87.1 & 91 & 34.1 \\
Small banks set & 81.1 & 97.8 & 151.1 & 70.4 \\
\hline
\end{tabular}

References: results of this study.

TABLE 10: The concentration degree of the Iranian banking industry.

\begin{tabular}{lc}
\hline Year & The concentration degree of the Iranian banking industry based on the Herfindahl-Hirschman index (deposits viewpoint) \\
\hline 2010 & 1165.5 \\
2011 & 1125.2 \\
2012 & 1064.32 \\
2013 & 1071.3 \\
2014 & 981.2 \\
2015 & 982.6 \\
2016 & 945.5 \\
2017 & 872.1 \\
2018 & 844.3 \\
\hline
\end{tabular}

References: results of this study.

TABLE 11: The change dispersion of deposits share, PINPADs, and POSs.

\begin{tabular}{lccc}
\hline Year & Variation coefficient of POS & Variation coefficient of PINPADs & Variation coefficient of deposits share \\
\hline 2010 & 0.023 & 0.042 & 0.100 \\
2011 & 0.021 & 0.042 & 0.054 \\
2012 & 0.0188 & 0.048 & 0.038 \\
2013 & 0.019 & 0.044 & 0.028 \\
2014 & 0.016 & 0.039 & 0.029 \\
2015 & 0.0161 & 0.039 & 0.024 \\
2016 & 0.023 & 0.042 & 0.100 \\
2017 & 0.021 & 0.042 & 0.054 \\
2018 & 0.0188 & 0.048 & 0.038 \\
\hline
\end{tabular}

References: results of this study.

TABLE 12: Average dispersion growth of deposit shares, PINPADs, and POSs.

\begin{tabular}{|c|c|}
\hline $\begin{array}{l}\text { Average annual growth of variation coefficient of deposit } \\
\text { share }(\%)\end{array}$ & -72.7 \\
\hline $\begin{array}{l}\text { Average annual growth of variation coefficient of } \\
\text { PINPADs (\%) }\end{array}$ & -51.3 \\
\hline Average annual growth of variation coefficient of POSs (\%) & -95.3 \\
\hline
\end{tabular}

4.3. The Concentration Degree of the Iranian Banking Sector. As stated above, in order to ensure the accuracy and inconsistency of the conclusion that e-banking has reduced the concentration degree in the Iranian banking industry, with the reality of the degree of concentration in the industry, the concentration degree of the Iranian banking industry has been obtained during the years of 2010-2018. The results are listed in Table 10 after the necessary calculations.

According to the Herfindahl-Hirschman Index, by observing how industries are competitive or noncompetitive, it is clear that the banking industry is experiencing a decline in concentration and the industry is slowly moving from a tight oligopoly to a loose oligopoly.
4.4. The Results Analysis. The analysis of the reasons for the decline in concentration states that the share of banks has decreased due to the increase in the share of small banks. On the other hand, the estimation of the model shows that there is a positive relationship between the use of electronic banking and the share of banks. It has been found that as the small banks have strongly utilized e-banking, it would result in increasing their share, thereby reducing the concentration degree. As such, it can be concluded that e-banking has reduced the concentration degree in Iran. As can be observed from Table 11, as the process of distribution of the share of deposits (shown in the second column) improves, the process of PINPAD distribution and the distribution of POSs (in the third and fourth columns) can also be improved. In other words, their dispersion is decreasing.

This concept is better understood in Table 12. As shown in Table 12, the average dispersion growth of deposit data (variation coefficient) of deposit data, PINPADs, and POSs is strongly decreasing.

The results obtained using our proposed method are the same as the results in [21]. However, in our proposed method, uncertainty is also considered. 


\section{Conclusion}

In the Iranian banking sector, all banks utilize e-banking. To summarize the effect of e-banking on the concentration degree of the Iranian banking sector, or to examine how the relative size of banks changes with respect to e-banking, in addition to investigating the impact of e-banking on banks' share, a hybrid artificial neural network model-fuzzy regression and descriptive statistics have also been employed. This is because it was necessary to determine which increase or decrease in the banks' share was in favor of the banks in order to discuss increasing or decreasing the concentration degree. Thus, the concentration degree on Iran's banking sector has reduced.

The analysis of the reasons for the decline in concentration indicates that the share of banks has decreased due to an increase in the share of small banks. On the other hand, the estimation of the model shows that there is a positive relationship between the use of electronic banking and the share of banks. It was also found that small banks have more strongly employed e-banking, thereby increasing their share and thus reducing the concentration degree. Therefore, it can be concluded that e-banking has reduced the concentration degree. An examination of the concentration degree on the Iranian banking industry is also in accordance with this conclusion.

\section{Data Availability}

The numerical data used to support the findings of this study are included within the article.

\section{Conflicts of Interest}

The authors declare that they have no known competing financial interests or personal relationships that could have appeared to influence the work reported in this paper.

\section{References}

[1] F. Khodadad Kashi and P. Jafari, "Evaluating the effects of structure on Iranian banking industry," Journal of Economic Research, vol. 47, no. 1, pp. 71-91, 2012.

[2] M. Kato and Y. Honjo, "Market share instability and the dynamics of competition: a panel data analysis of Japanese manufacturing industries," Review of Industrial Organization, vol. 28, no. 2, pp. 165-182, 2006.

[3] A. Souri, Econometric, Farhang Shenasi Publications, Tehran, Iran, 7th edition, 2009.

[4] M. Khashei, "Forecasting and analysis of Esfahan steel company productions price in Tehran metals exchangeusing arti cial neural networks," Master of Science thesis, Isfahan University of Technology, Isfahan, Iran, 2005.

[5] S. Kang, An investigation of the use of feedforward neural networks for forecasting, Ph.D. thesis, Kent StateUniversity, Kent, OH, USA, 1991.

[6] H. Tanaka, "Fuzzy data analysis by possibilistic linear models," Fuzzy Sets and Systems, vol. 24, no. 3, pp. 363-375, 1987.
[7] H. Tanaka and H. Ishibuchi, "Possibility regression analysis based on linear programming," in Fuzzy Regression Analysis, pp. 47-70, Physica-Verlag, Heidelberg, Germany, 1992.

[8] Q. Song and B. S. Chissom, "Forecasting enrollments with fuzzy time series-part I," Fuzzy Sets and Systems, vol. 54, no. 1, pp. 1-9, 1993.

[9] S.-M. Chen, "Forecasting enrollments based on fuzzy time series," Fuzzy Sets and Systems, vol. 81, no. 3, pp. 311-319, 1996.

[10] J. M. Bates and C. W. J. Granger, "The combination of forecasts," Operational Research Society, vol. 20, no. 4, pp. 451-468, 1969.

[11] R. T. Clemen, "Combining forecasts: a review and annotated bibliography," International Journal of Forecasting, vol. 5, no. 4, pp. 559-583, 1989.

[12] D. J. Reid, "Combining three estimates of gross domestic product," Economica, vol. 35, no. 140, pp. 431-444, 1968.

[13] E. Cadenas and W. Rivera, "Wind speed forecasting in three different regions of Mexico, using a hybrid ARIMA-ANN model," Renewable Energy, vol. 35, no. 12, pp. 2732-2738, 2010.

[14] M.-Y. Cheng, H.-C. Tsai, and E. Sudjono, "Evolutionary fuzzy hybrid neural network for project cash flow control," Engineering Applications of Artificial Intelligence, vol. 23, no. 4, pp. 604-613, 2010.

[15] F. H. Basturk, "Characteristics and competition structure of Turkish insurance industry," Procedia-Social and Behavioral Sciences, vol. 62, pp. 1084-1088, 2012.

[16] M. Khashei, M. Bijari, and G. A. Raissi Ardali, "Improvement of auto-regressive integrated moving average models using fuzzy logic and artificial neural networks (ANNs)," Neurocomputing, vol. 72, no. 4-6, pp. 956-967, 2009.

[17] L. Yu, S. Wang, and K. K. Lai, "A novel nonlinear ensemble forecasting model incorporating GLAR and ANN for foreign exchange rates," Computers \& Operations Research, vol. 32, no. 10, pp. 2523-2541, 2005.

[18] R. Fildes and S. Makridakis, "The impact of empirical accuracy studies on time series analysis and forecasting," International Statistical Review/Revue Internationale de Statistique, vol. 63, no. 3, pp. 289-308, 1995.

[19] A. F. Shapiro, "Fuzzy regression and the term structure of interest rate," in Proceedings of the 14th International AFIR Colloquium, pp. 29-45, Boston, MA, USA, April 2004.

[20] H. F. Wang and R. C. Tsaur, "Insight of a fuzzy regression model," Fuzzy Set and Systems, vol. 112, no. 3, pp. 355-369, 2000.

[21] H. S. Shamsedin and N. Dargazani, "The effect of electronic banking on deposit attraction and market concentration in Iran's banking industry, Iran," Economic Review, vol. 24, no. 2, pp. $545-565,2020$. 